\title{
Association of Serum LDL Cholesterol Level with Periodontitis among Patients Visiting a Tertiary-care Hospital
}

\author{
Sharma S ${ }^{1}$, Lamsal $M^{2}$, Sharma $S^{3}$, Niraula $\mathrm{SR}^{4}$ Koirala $\mathrm{B}^{5}$ \\ 'Department of Periodontics, ${ }^{2}$ Department of Biochemistry, ${ }^{3}$ Department of Internal Medicine, ${ }^{4}$ Department of Community
} Medicine, ${ }^{5}$ College of Dental Surgery, B.P. Koirala Institute of Health Sciences, Dharan, Nepal

\section{ABSTRACT}

Introduction: High low-density lipoproteins (LDL) cholesterol is one of the major risk factors for cardiovascular disease. In recent years, some evidence has been presented that periodontitis, an infectious inflammatory condition of the periodontium, is associated with an increased risk of cardiovascular disease. To further elucidate this association, we have studied the levels of LDL cholesterol, a known risk marker for cardiovascular disease, in a periodontally-diseased group.

Methods: The levels of serum LDL cholesterol in 47 subjects with mild to severe (clinical attachment loss equal to or greater than $1 \mathrm{~mm}$ ) chronic generalized (at least $30 \%$ of teeth affected) periodontitis with the mean age of $42.21 \pm 1.46$ years were measured and compared with those obtained from 42 age $(39.83 \pm 0.94)$ and sex matched controls. Both groups were free from systemic illnesses.

Results: The mean serum LDL cholesterol in periodontitis patients was found to be significantly higher $(\mathrm{P}<0.001)$ as compared to that of the controls. The mean clinical attachment loss was positively correlated with serum LDL cholesterol $(\mathrm{P}<0.01)$ and gingival index $(\mathrm{P}<0.05)$. The frequency of persons with pathologic values of LDL cholesterol was significantly higher in periodontitis patients compared with that of the controls.

Conclusions: These results showed that high serum LDL cholesterol may be associated with periodontitis in healthy people. However, it is unclear whether periodontitis causes an increase in the levels of serum LDL or an increased LDL is a risk factor for both periodontitis and cardiovascular disease.

Keywords: Cardiovascular disease, LDL cholesterol, periodontitis.

\section{INTRODUCTION}

Cardiovascular disease (CVD) is one of the major causes of mortality worldwide ${ }^{1}$ and the leading cause of death in Europe and the US. ${ }^{2}$ Atherosclerotic vascular disease encompasses coronary heart disease, cerebrovascular disease and peripheral artery disease, and is responsible for the majority of cases of CVD and stroke. ${ }^{3} \mathrm{~A}$ number of common infectious inflammatory conditions, including periodontitis, have been hypothesized to promote atherogenesis and increase the risk for cardiovascular and cerebrovascular events. . $^{4-6}$
Periodontitis is an inflammatory disease of the supporting tissues of the teeth caused by microorganisms resulting in progressive destruction of the periodontal ligament and alveolar bone with pocket formation, recession, or both. It is classified as mild, moderate, and severe, based on the severity of destruction of the periodontium. ${ }^{7}$

\footnotetext{
Correspondence:

Dr. Shivalal Sharma

College of Dental Surgery

B.P.Koirala Institute of Health Sciences

Dharan, Nepal

Mobile: 9842064953

E-mail: drshivalalsharma@yahoo.co.uk
} 
Generally, it has been assumed that atherosclerotic plaques form as a result of the accumulation of lowdensity lipoprotein (LDL) cholesterol in the arterial wall. However, it has also been noted that the formation of the lesion is due to a complex series of events, reminiscent of an inflammatory reaction. ${ }^{8}$ The origin of this inflammatory reaction has lead to the consideration that the infection-like periodontitis may be a component of the mechanism that results in the development of this lesion.

The aim of the study was to compare the levels of LDL cholesterol in patients with periodontitis, and periodontally-healthy subjects to evaluate the association between the two, if any.

\section{METHODS}

This is a crossectional observational study done in the Department of Periodontology, College of Dental Surgery (CODS), B.P. Koirala Institute of Health Sciences (BPKIHS), Dharan, Nepal. Healthy subjects, aged between 25 - 60 years, willing to participate in the study, presenting with mild to severe (clinical attachment loss equal to or greater than $1 \mathrm{~mm}$ ), generalized (at least $30 \%$ of teeth affected) periodontitis ${ }^{7}$ were included in the study (Periodontitis group). Participant of the Control group were age and sex matched healthy subjects without gingivitis or periodontitis. All patients gave written informed consent. The study had been reviewed and approved by the Institutional Ethical Review Board of the BPKIHS.

Exclusion criteria for the Periodontitis group included: (i) known systemic diseases, (ii) recent history and/or presence of other infections, (iii) systemic antibiotic treatment in the preceding three months, (iv) any concomitant medical therapy, (v) pregnancy, (vi) obese individuals (BMI >30), (vii) smoking or tobacco use in any form, and (viii) presence of $<16$ teeth. Exclusion criteria for the Control group included all the exclusion criteria of the Periodontitis group plus the presence of gingivitis and/or periodontitis.
At the first visit, a detailed medical history and general as well as oral examinations were carried out on all patients and control subjects by an examiner. The plaque index ${ }^{9}$, gingival index ${ }^{10}$, and clinical attachment loss $^{7}$ were recorded, and then the patients were sent for fasting blood sampling for LDL cholesterol. A total of 95 individuals met the inclusion criteria. Eighty nine subjects were enrolled in the study; the remaining six subjects did not give consent. Of the 89 subjects enrolled in the study, 47 (25 females and 22 males) were of the Periodontitis group, and the remaining 42 (22 females and 20 males) were of the Control group.

To measure the standard clinical periodontal parameters (plaque detection, bleeding on probing, and clinical attachment loss), a mouth mirror, an explorer, and a University of North Carolina-15 (UNC-15) probe was used.

Measurement of fasting serum LDL cholesterol was done in the Department of Biochemistry, BPKIHS, Dharan, using the routine enzymatic method. In order to identify subjects with pathological values, a serum LDL cholesterol of $>160 \mathrm{mg} / \mathrm{dl}$ was used as the cut-off point according to the laboratory's recommendation.

SPSS Ver. 11.0 was used to analyze the data. The equality of means of different variables was tested by using the t-test. The association between the variables was examined with Pearson Correlation Coefficient. The level of significance was set up at $5 \%$.

\section{RESULTS}

The mean ages among the two groups were found to be statistically insignificant, which shows that the age distribution among the groups was homogenous. The mean serum LDL cholesterol in periodontitis subjects was found to be significantly higher $(P<0.001)$ compared to the controls (Table 1 ).

Table 1. Mean Age and LDL-Cholesterol Distribution among the Groups.

\begin{tabular}{llllllll}
\hline Variables & Group & $\mathrm{N}$ & Mean & $\begin{array}{l}\text { Std. } \\
\text { Deviation }\end{array}$ & $\begin{array}{l}\text { Std. Error } \\
\text { Mean }\end{array}$ & t-value & P value \\
\hline \multirow{2}{*}{ Age (Yrs.) } & Periodontitis & 47 & 42.21 & 10.01 & 1.46 & 1.33 & 0.186 \\
& Control & 42 & 39.83 & 6.12 & 0.94 & $<.001$ \\
LDL & Periodontitis & 47 & 116.62 & 35.96 & 5.24 & 4.43 & $<0.0015$ \\
Cholesterol & Control & 42 & 84.98 & 30.80 & 4.75 & & \\
& & & & & & &
\end{tabular}

$\mathrm{P}<0.05$ is significant. 
The mean clinical attachment loss was positively correlated with serum LDL cholesterol $(P<0.01)$, and gingival index $(\mathrm{P}<0.05)$, but there was no significant association of age and plaque index with mean clinical attachment loss (Table 2). However, the gingival index did not correlate with either the serum LDL cholesterol or plaque index. Similarly, the plaque index had no association with the age and LDL cholesterol.

In four subjects among the periodontitis group and in one control subject, the LDL cholesterol value was in the pathologic range.

\section{Table 2. Correlation Matrix of Variables under Study}

\begin{tabular}{|c|c|c|c|c|c|c|}
\hline & & AGE & LDL CHOL & $\mathrm{PI}$ & GI & MEAN CAL \\
\hline \multirow[t]{2}{*}{ AGE } & Corr. Coeff. & 1 & -0.088 & -0.084 & -0.214 & 0.048 \\
\hline & $P$ value & . & 0.556 & 0.577 & 0.149 & 0.749 \\
\hline \multirow[t]{2}{*}{ LDL CHOL } & Corr. Coeff. & - 0.088 & 1 & 0.096 & 0.213 & $0.395 * *$ \\
\hline & $P$ value & 0.556 & . & 0.521 & 0.151 & 0.006 \\
\hline \multirow[t]{2}{*}{ PI } & Corr. Coeff. & -0.084 & 0.096 & 1 & 0.251 & -0.04 \\
\hline & $\mathrm{P}$ value & 0.577 & 0.521 & . & 0.089 & 0.791 \\
\hline \multirow[t]{2}{*}{$\mathrm{GI}$} & Corr. Coeff. & -0.214 & 0.213 & 0.251 & 1 & $0.293 *$ \\
\hline & $\mathrm{P}$ value & 0.149 & 0.151 & 0.089 & . & 0.045 \\
\hline \multirow[t]{2}{*}{$\begin{array}{l}\text { MEAN } \\
\text { CAL }\end{array}$} & Corr. Coeff. & 0.048 & $0.395 * *$ & -0.04 & $0.293^{*}$ & 1 \\
\hline & $\mathrm{P}$ value & 0.749 & 0.006 & 0.791 & 0.045 & . \\
\hline $\mathrm{N}$ & 47 & 47 & 47 & 47 & 47 & 47 \\
\hline
\end{tabular}

** Correlation is significant at the 0.01 level (2-tailed), * Correlationis signnificant at the 0.05 level (2-tailed)

$\mathrm{PI}$, plaque Index; GI, gingival index; CAL, clinical attachment Loss

\section{DISCUSSION}

For over more than five decades, the prevailing view among dentists and physicians was that periodontal infections were localized only to the marginal periodontium (gingiva and its associated structures) and that they rarely had systemic implications in healthy individuals. More recent evidence, however, has indicated that patients with periodontitis present with increased systemic inflammation, as indicated by raised serum levels of various inflammatory markers when compared with those in unaffected control populations. ${ }^{11-13}$ Further, higher concentrations of total and LDL cholesterol and triglycerides and lower concentrations of high density lipoprotein (HDL) cholesterol have been observed in individuals with

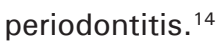

Katz et al. conducted a study on the association between hypercholesterolaemia, cardiovascular disease, and severe periodontal disease and found a significant association of patients with hypercholesterolaemia and periodontitis. ${ }^{15}$

In our study, patients with periodontitis had a significantly higher levels of serum LDL cholesterol compared to a periodontally-healthy control group. Our findings are in agreement with Moeintaghvi et al, who also found that the presence of periodontitis was significantly associated with elevated serum LDL cholesterol levels. ${ }^{16}$ In addition, our result is also in agreement with Joshi and Marawar ${ }^{17}$ and Losche et al. ${ }^{18}$

We also tried to correlate the LDL cholesterol levels with different periodontal parameters, where it was seen that the mean clinical attachment loss was positively correlated with serum LDL cholesterol levels $(P<0.01)$, and gingival index $(P<0.05)$. In most of 
our cases, it was seen that the greater the attachment loss, the higher was the LDL cholesterol levels and this is in accordance with the study of D'Auito et al. 19 However, there was no significant association of age and plaque index with mean clinical attachment loss. There was also no association between serum LDL cholesterol levels and gingival index (severity of gingival inflammation). The most probable reason for this could be the examiner's over-emphasis on measure of loss of attachment, which is more the diagnostic feature of periodontitis than the degree of gingival inflammation.

The frequency of pathological LDL cholesterol levels in our study was more prevalent in the patients than in the controls, and is in agreement with Moeintaghvi et al., ${ }^{16}$ The cut-off point for pathological value used for LDL in Moeintaghvi's ${ }^{16}$ study was: LDL > $190 \mathrm{mg} / \mathrm{dl}$, and our laboratory's was LDL > $160 \mathrm{mg} / \mathrm{dl}$.

These results, however, do not allow for generalization to periodontal patients suffering from less severe and/ or more localized forms of the disease. Data will have to be confirmed with expansion in larger population if we are to better understand what proportion of patients suffering from severe periodontitis has increased systemic inflammation as a result of this chronic infection. ${ }^{20}$

It is difficult to explain the association between raised LDL and periodontitis. One of the plausible mechanisms is that there is a direct effect of periodontal infections on atherogenesis and/or progression of atherosclerosis could be mediated through translocation of periodontal pathogens from the oral cavity into the blood circulation. Indeed, bacteremia has been reported to occur not only after episodes of active periodontal therapy ${ }^{21}$, but also after relatively minor soft tissue manipulation such as periodontal probing. ${ }^{22}$ However, its frequency of occurrence may be lower than previously considered. ${ }^{23}$ Nevertheless, circulating bacteria and/or bacterial products can conceivably reach distant sites as demonstrated by Haraszthy et al., ${ }^{24}$ On the other hand, bacterial toxins (LPS) can also induce changes in cholesterol concentrations ${ }^{25}$ (reduced HDL and increased LDL). ${ }^{26}$
The proposed indirect mechanisms by which periodontal infection may contribute to the pathogenesis of atherosclerotic vascular disease originate on the fact that there is an abundant production of inflammatory mediators (IL-1, TNF- $\alpha$ ) locally in the periodontal lesion, which may subsequently enter the systemic circulation and produce its effect on other systemic mediators (IL6) which might induce alterations of lipid metabolism such as increased LDL cholesterol due to increased hepatic lipogenesis, lipolysis from adipose tissue, or reduced blood clearance. ${ }^{27}$ It is observed that proinflammatory cytokines are found to be elevated in the serum of patients with periodontitis, ${ }^{28}$ and these mediators have traditionally been considered as markers of cardiovascular disease. ${ }^{3,5,28}$

On the other hand, a possible role of hyperlipidemia for periodontitis is also obvious from several studies. Hyperlipidemia is known to cause hyperactivity of white blood cells. ${ }^{29,30}$ Hyperactivity of white cells, e.g., increased production of oxygen radicals, has also been shown to be frequently associated with progressive periodontitis in adults. ${ }^{31,32}$

Although the control subjects were matched for age and sex, the life style and socioeconomic factors such as diet and physical activity were not taken in account that could have altered the outcome of the study. This is the limitation of our study.

\section{CONCLUSIONS}

The results showed that patients with periodontitis had significantly higher levels of serum LDL cholesterol than subjects with normal periodontium. However, it is not yet clear whether the increased LDL cholesterol observed in periodontitis patients is the cause or the consequence of periodontitis. Therefore, unless and until definitive clinical studies can demonstrate or further explain the associations between periodontitis and cardiovascular disease, it is premature to counsel our patients to 'brush or die'. 


\section{REFERENCES}

1. World Health Organization, Geneva. World Health Statistics 2006. Geneva: WHO; 2006.

2. Bethesda. Morbidity \& Mortality: Cardiovascular, Lung, and Blood Disease. US: National Heart, Lung and Blood Institute, National Institute of Health; 2004.

3. Hackam DG, Anand SS. Emerging risk factors for atherosclerotic vascular disease: a critical review of the evidence. JAMA. 2003;290:932-40.

4. L Nibali, D'Aiuto F, Griffiths G, Patel K, Suvan J, Tonetti MS. Severe periodontitis is associated with systemic inflammation and a dysmetabolic status: a case - control study. J Clin Periodontal. 2007;34:931-7.

5. Sanz M, D'Aiuto F, Deanfield J, Aviles FF. European workshop in periodontal health and cardiovascular diseasescientific evidence on the association between periodontal and cardiovascular diseases: a review of the literature. European Heart Journal Suppliments. 2010;12(suppl B): B3B12.

6. Ana P, Ljiljana K, Zlata B, Zoran P, Dimitrije M. Effect of Periodontal Treatment on Lipoproteins Levels in Plasma in Patients with Periodontitis. Southern Medical Journal. 2011;104(8): 547-52.

7. Novak MJ, Novak KF: Chronic Periodontitis. In: Newman MG, Takei HH, Klokkevold PR and Carranza FA: Clinical Periodontology. 10th edition. US: Saunders; 2006.p. 494-9

8. Ross R. Atherosclerosis - An inflammatory disease. NEJM. 1999;340:115-26

9. Silness P, Loe H. Periodontal disease in pregnancy. Acta Odontol Scand. 1964;22:121.

10. Loe H, Silness P. Periodontal disease in pregnancy. Acta Odontol Scand. 1963;21:533.

11. Duan JY, Ou-Yang XY, Zhou XY . Effect of periodontal initial therapy on the serum level of lipid in the patients with both periodontitis and hyperlipidemia. Beijing Da Xue Xue Bao . 2009;41(1):36-9.

12. Taleghani F, Shamaei M, Shamaei M. Association between chronic periodontitis and serum lipid levels. Acta Medica Iranica. 2010;48(1):47-50.

13. Ramesh A, Shaju JP, Jade RM. Association between chronic generalized periodontitis and hyperlipidemia - a case control study. Bangladesh Journal of Medical Science. 2010;09(2):95100.

14. Perera WKL, Amaratunga EAAP, Mohoty WC, Perera NPM, Kularatne S, Sivakanesan R et al. Association between Plasma Lipid Levels and Periodontitis: A Preliminary Analysis. Proceedings of the Peradeniya University Research Sessions, Sri Lanka. Vol. 16, 24 $4^{\text {th }}$ November 2011; 62.

15. Katz J, Chaushu G, Sharab Y. On the association between hypercholesterolemia, cardiovascular disease and severe periodontal disease. J Clin Periodontol. 2001;28:865-8.

16. Moeintaghvi A, Ardakani AH, Ardakani MT, Tabatabaie I. Hyperlipidemia in patients with periodontitis. J Contemp Dent Pract 2005;15:6(3):78-85.
17. Joshi NV, Marawar PP. Hyperlipidemia- A Link Between Periodontitis and Coronary Heart Disease. J Indian Dent Assoc. 2011;5(2):183-6.

18. Losche W, Karapetow F, Pohl A. Plasma lipid and blood glucose levels in patients with destructive periodontal disease. J Clin Periodontol. 2000;27:537-41.

19. D'Aiuto F, Parkar M, Andreou G, Suvan J, Brett PM, Ready $\mathrm{D}$, et al. Periodontitis and systemic inflammation: Control of the local infection is associated with a reduction in serum inflammatory markers. J Dent Res 2004;83:156-60.

20. Papapanou PN. Periodontal diseases: Epidemiology. Ann Periodontol. 1996;1:1-36.

21. Baltch AL, Schaffer C, Hammer MC. Bacteremia following dental cleaning in patients with and without penicillin prophylaxis. Am Heart J. 1982;104:1335-9.

22. Daly C, Mitchell D, Grossberg D. Bacteraemia caused by periodontal probing. Aust Dent J. 1997;42:77-80.

23. Kinane DF, Riggio MP, Walker KF. Bacteraemia following periodontal procedures. J Clin Periodontol. 2005;32:708-13.

24. Haraszthy VI, Zambon JJ, Trevisan M. Identification of periodontal pathogens in atheromatous plaques. J Periodontol. 2000;71:1554-60.

25. Uchiumi D, Kobayashi M, Tachikawa T, Hasegawa K. Subcutaneous and continuous administration of lipopolysaccharide increases serum levels of triglyceride and monocyte chemoattractant protein-1 in rats. J Periodontal Res. 2004;39:120-8.

26. Pussinen PJ, Jauhiainen M, Vilkuna-Rautiainen T, Sundvall J, Vesanen M, Mattila K, et al. Periodontitis decreases the antiatherogenic potency of high density lipoprotein. J Lipid Res. 2004;45:139-47.

27. Iacopino AM, Cutler CW. Pathophysiological relationships between periodontitis and systemic disease: Recent concepts involving serum lipids. J Periodontol. 2000;71:1375-84.

28. Vaish S, Menon I. Periodontitis and Cardiovascular Diseases - A Review on causality hypotheses. International Journal of Public Health Dentistry. 2011: 2(1):13-8.

29. Croft KD, Beikin LJ, Vandogen R. Leukocyte and platelet function and eicosanoid production in subjects with hypercholesterolemia. Atherosclerosis. 1990;83:101-8.

30. Krause S, Pohl A, Pohl C. Increased generation of reactive oxygen species in mononuclear cells from hypercholesterolaemic patients. Thrombosis Res. 1993;71:237-40.

31. Shapira L, Borinski R, Sela MN. Super oxide formation and chemiluminescence of peripheral polymorphonuclear leukocytes in rapidly progressive periodontitis patients. J Clin Periodontol. 1991;18:44-8.

32. Gustafsson A, Asman B. Increased release of free oxygen radicals from peripheral neutrophils in adult periodontitis after FC- receptor stimulation. J Clin Periodontol. 1996;23:3844. 